\title{
Lower Bounds of Dirichlet Eigenvalues for General Grushin Type Bi-Subelliptic Operators
}

\author{
Hua Chen*, Hongge Chen, Junfang Wang and Nana Zhang \\ School of Mathematics and Statistics, Wuhan University, Wuhan 430072, China
}

Received 21 August 2017; Accepted (in revised version) 4 October 2017

\begin{abstract}
Let $\Omega$ be a bounded open domain in $\mathbb{R}^{n}$ with smooth boundary $\partial \Omega$. Let $X=\left(X_{1}, X_{2}, \cdots, X_{m}\right)$ be a system of general Grushin type vector fields defined on $\Omega$ and the boundary $\partial \Omega$ is non-characteristic for $X$. For $\Delta_{X}=\sum_{j=1}^{m} X_{j}^{2}$, we denote $\lambda_{k}$ as the $k$-th eigenvalue for the bi-subelliptic operator $\Delta_{X}^{2}$ on $\Omega$. In this paper, by using the sharp sub-elliptic estimates and maximally hypoelliptic estimates, we give the optimal lower bound estimates of $\lambda_{k}$ for the operator $\Delta_{X}^{2}$.
\end{abstract}

Key Words: Eigenvalues, degenerate elliptic operators, sub-elliptic estimate, maximally hypoelliptic estimate, bi-subelliptic operator.

AMS Subject Classifications: 35J30, 35J70, 35P15

\section{Introduction and main results}

Let $X=\left(X_{1}, X_{2}, \cdots, X_{m}\right)$ be the system of general Grushin type vector fields, which is defined on an open domain $W$ in $\mathbb{R}^{n}(n \geq 2)$.

Let $J=\left(j_{1}, \cdots, j_{k}\right), 1 \leq j_{i} \leq m$ be a multi-index, $X^{J}=X_{j_{1}} X_{j_{2}} \cdots X_{j_{k}}$, we denote $|J|=k$ be the length of $J$, if $|J|=0$, then $X^{J}=i d$. We introduce following function space (cf. $\left.[18,21,23]\right)$ :

$$
H_{X}^{2}(W)=\left\{u \in L^{2}(W)\left|X^{J} u \in L^{2}(W),\right| J \mid \leq 2\right\} .
$$

It is well known that $H_{X}^{2}(W)$ is a Hilbert space with norm $\|u\|_{H_{X}^{2}(W)}^{2}=\sum_{|J| \leq 2}\left\|X^{J} u\right\|_{L^{2}(W)}^{2}$.

Assume the vector fields $X=\left(X_{1}, X_{2}, \cdots, X_{m}\right)$ satisfy Hörmander's condition :

Definition 1.1 (cf. $[2,12])$. We say that $X=\left(X_{1}, X_{2}, \cdots, X_{m}\right)$ satisfies the Hörmander's condition in $W$ if there exists a positive integer $Q$, such that for any $|J|=k \leq Q, X$ together with all $k$-th repeated commutators

$$
X_{J}=\left[X_{j_{1}},\left[X_{j_{2}},\left[X_{j_{3}}, \cdots,\left[X_{j_{k-1}}, X_{j_{k}}\right] \cdots\right]\right]\right]
$$

${ }^{*}$ Corresponding author. Email addresses: chenhua@whu.edu.cn (H. Chen), hongge_chen@whu.edu.cn (H. G. Chen), wangjunfang@whu.edu.cn (J. F. Wang), zhangnana@whu.edu.cn (N. N. Zhang) 
span the tangent space at each point of $W$. Here $Q$ is called the Hörmander index of $X$ in $W$, which is defined as the smallest positive integer for the Hörmander's condition to be satisfied.

For any bounded open subset $\Omega \subset \subset W$, we define the subspace $H_{X, 0}^{2}(\Omega)$ to be the closure of $C_{0}^{\infty}(\Omega)$ in $H_{X}^{2}(W)$. Since $\partial \Omega$ is smooth and non characteristic for $X$, we know that $H_{X, 0}^{2}(\Omega)$ is well defined and also a Hilbert space. In this case, we also say that $X$ satisfies the Hörmander's condition on $\Omega$ with Hörmander index $1 \leq Q<+\infty$. Thus $X$ is a finitely degenerate system of vector fields on $\Omega$ and the finitely degenerate elliptic operator $\Delta_{X}=\sum_{i=1}^{m} X_{i}^{2}$ is a sub-elliptic operator.

The degenerate elliptic operator $\Delta_{X}$ has been studied by many authors, e.g., Hörmander [11], Jerison and Sánchez-Calle [13], Métivier [17], Xu [23]. More results for degenerate elliptic operators can be found in $[2-6]$ and $[9,10,12,14]$.

In this paper, we study the following eigenvalues problem for bi-subelliptic operators in $H_{X, 0}^{2}(\Omega)$ :

$$
\begin{cases}\Delta_{X}^{2} u=\lambda u & \text { in } \Omega, \\ u=0, X u=0 & \text { on } \partial \Omega,\end{cases}
$$

where $X$ will be the following general Grushin type vector fields (see (1.5) and (1.7) below). In this case we know that for each $j, X_{j}$ is formally skew-adjoint, i.e., $X_{j}^{*}=-X_{j}$. Then there exists a sequence of discrete eigenvalues $\left\{\lambda_{j}\right\}_{j \geq 1}$ for the problem (1.1), which satisfying $0<\lambda_{1} \leq \lambda_{2} \leq \lambda_{3} \leq \cdots \leq \lambda_{k} \cdots$ and $\lambda_{k} \rightarrow+\infty$ as $k \rightarrow+\infty$ (see Proposition 2.5 below).

In the classical case, if $X=\left(\partial_{x_{1}}, \cdots, \partial_{x_{n}}\right)$, then $\Delta_{X}^{2}=\Delta^{2}$ is the standard bi-harmonic operator. In this case our problem is motivated from the following classical clamped plate problem, namely

$$
\begin{cases}\Delta^{2} u=\lambda u & \text { in } \Omega, \\ u=\frac{\partial u}{\partial v}=0 & \text { on } \partial \Omega\end{cases}
$$

where $\Delta=\partial_{x_{1}}^{2}+\partial_{x_{2}}^{2}+\cdots+\partial_{x_{n}}^{2}, \frac{\partial u}{\partial v}$ denotes the derivative of $u$ with respect to the outer unit normal vector $v$ on $\partial \Omega$.

For the eigenvalues of the clamped plate problem (1.2), Agmon [1] and Pleijel [20] showed the following asymptotic formula

$$
\lambda_{k} \sim \frac{16 \pi^{4}}{\left(B_{n} \operatorname{vol}(\Omega)\right)^{\frac{4}{n}}} k^{\frac{4}{n}} \quad \text { as } \quad k \rightarrow+\infty,
$$

where $B_{n}$ denotes the volume of the unit ball in $R^{n}$. In 1985, Levine and Protter [15] proved that

$$
\frac{1}{k} \sum_{i=1}^{k} \lambda_{i} \geq \frac{n}{n+4} \frac{16 \pi^{4}}{\left(B_{n} \operatorname{vol}(\Omega)\right)^{\frac{4}{n}}} k^{\frac{4}{n}}
$$


Later in 2012, Cheng and Wei [7] showed that the eigenvalues of the bi-harmonic operator satisfy

$$
\begin{aligned}
\frac{1}{k} \sum_{i=1}^{k} \lambda_{i} \geq & \frac{n}{n+4} \frac{16 \pi^{4}}{\left(B_{n} \operatorname{vol}(\Omega)\right)^{\frac{4}{n}}} k^{\frac{4}{n}} \\
& +\left(\frac{n+2}{12(n+4)}-\frac{1}{1152 n^{2}(n+4)}\right) \frac{\operatorname{vol}(\Omega)}{I(\Omega)} \frac{n}{n+2} \frac{4 \pi^{4}}{\left(B_{n} \operatorname{vol}(\Omega)\right)^{\frac{2}{n}}} k^{\frac{2}{n}} \\
& +\left(\frac{1}{576 n(n+4)}-\frac{1}{27648 n^{2}(n+2)(n+4)}\right)\left(\frac{\operatorname{vol}(\Omega)}{I(\Omega)}\right)^{2},
\end{aligned}
$$

where $I(\Omega)$ is the moment of inertia of $\Omega$.

Next, we consider the situation for the bi-subelliptic operators $\Delta_{X}^{2}$. Before we state our results, we need the following concepts:

Definition 1.2. If $X$ satisfies the Hörmander's condition in $W$ with the Hörmander index $Q \geq 1$. Then for each $1 \leq j \leq Q$ and $x \in W$, we denote $V_{j}(x)$ as the subspace of the tangent space $T_{x}(W)$ spanned by the vector fields $X_{J}$ with $|J| \leq j$. We say the system of the vector fields $X$ satisfies Métivier's condition on $\Omega$ if the dimension of $V_{j}(x)$ is constant $v_{j}$ in a neighborhood of each $x \in \bar{\Omega}$, and in this case the Métivier index is defined as

$$
v=\sum_{j=1}^{Q} j\left(v_{j}-v_{j-1}\right), \quad \text { here } v_{0}=0 .
$$

As it well-known that under the Métivier's condition, we can get the asymptotic estimate for the eigenvalues of sub-elliptic operator $-\Delta_{X}$ (cf. [17]). However, for most degenerate vector fields $X$, the Métivier's condition will be not satisfied. Thus we need to introduce the following generalized Métivier index.

Definition 1.3. If $X$ satisfies the Hörmander's condition in $W$ with the Hörmander index $Q \geq 1$. Then for each $1 \leq j \leq Q$ and $x \in W$, we denote $V_{j}(x)$ as the subspace of the tangent space $T_{x}(W)$ spanned by the vector fields $X_{J}$ with $|J| \leq j$. We denote that

$$
v(x)=\sum_{j=1}^{Q} j\left(v_{j}(x)-v_{j-1}(x)\right), \quad \text { with } v_{0}(x)=0,
$$

where $v_{j}(x)$ is the dimension of $V_{j}(x)$. Then we define

$$
\widetilde{v}=\max _{x \in \bar{\Omega}} v(x),
$$

as the generalized Métivier index. It is obvious that $\tilde{v}=v$ if $X$ satisfies the Métivier's condition on $\Omega$. 
Recently, in case of $X$ to be some special Grushin vector fields Chen and Zhou [8] obtained lower bound estimates of eigenvalues for the bi-subelliptic operator $\Delta_{X}^{2}$. In this paper, we shall study the similar problem for more general Grushin type vector fields $X$. In the first part of this paper, we shall study the bi-subelliptic operators $\Delta_{X}^{2}$ in case of

$$
X=\left(\partial_{x_{1}}, \cdots, \partial_{x_{n-1}}, f(\bar{x}) \partial_{x_{n}}\right),
$$

where $f(\bar{x})=\sum_{|\alpha| \leq s} a_{\alpha} \bar{x}^{\alpha}$ is a multivariate polynomial of $\bar{x}$ with order $s, \bar{x}=\left(x_{1}, \cdots, x_{n-1}\right)$, $\alpha=\left(\alpha_{1}, \cdots, \alpha_{n-1}\right) \in Z_{+}^{n-1},|\alpha|=\alpha_{1}+\cdots+\alpha_{n-1}, a_{\alpha}$ are constants. We suppose that

$\left(H_{1}\right)$ : If $f(\bar{x})$ has a unique zero point at origin $\bar{x}=0$ in $\Omega$ only, and there exists a unique multi-index $\alpha_{0}$ with $\left|\alpha_{0}\right|=s_{0} \leq s$, satisfying $\left.\partial_{\bar{x}}^{\alpha_{0}} f(\bar{x})\right|_{\bar{x}=0} \neq 0$ and $\left.\partial_{\bar{x}}^{\alpha} f(\bar{x})\right|_{\bar{x}=0}=0$ for any $|\alpha|<\left|\alpha_{0}\right|$.

Thus we have the following result.

Theorem 1.1. Let $X=\left(\partial_{x_{1}}, \cdots, \partial_{x_{n-1}}, f(\bar{x}) \partial_{x_{n}}\right), \bar{x}=\left(x_{1}, x_{2}, \cdots, x_{n-1}\right)$. Under the condition $\left(H_{1}\right)$ above, $X$ satisfies the Hörmander's condition with its Hörmander index $Q=s_{0}+1$, and the generalized Métivier index of $X$ is $\tilde{v}=Q+n-1$. Suppose $\lambda_{j}$ is the $j$-th eigenvalue of the problem (1.1), then for all $k \geq 1$,

$$
\sum_{j=1}^{k} \lambda_{j} \geq \bar{C}(Q) k^{1+\frac{4}{\bar{v}}}-\frac{C_{2}(Q)}{C_{1}(Q)} k
$$

where

$$
\bar{C}(Q)=\frac{A_{Q}}{C_{1}(Q) n^{2}(n+Q+3)}\left(\frac{(2 \pi)^{n}}{Q \omega_{n-1}|\Omega|_{n}}\right)^{\frac{4}{n+Q-1}}(n+Q-1)^{\frac{n+Q+3}{n+Q-1}},
$$

and

$$
A_{Q}= \begin{cases}\min \left\{1, n^{\frac{3-Q}{2}}\right\}, & Q \geq 2, \\ n, & Q=1 .\end{cases}
$$

Here $C_{1}(Q), C_{2}(Q)$ are the constants in Proposition 2.3 below, $\omega_{n-1}$ is the area of the unit sphere in $\mathbb{R}^{n}$, and $|\Omega|_{n}$ is the volume of $\Omega$.

Remark 1.1. (1) Since $k \lambda_{k} \geq \sum_{j=1}^{k} \lambda_{j}$, then Theorem 1.1 shows that the eigenvalues $\lambda_{k}$ satisfy

$$
\lambda_{k} \geq \bar{C}(Q) k^{\frac{4}{\bar{v}}}-\frac{C_{2}(Q)}{C_{1}(Q)}, \quad \text { for all } \quad k \geq 1
$$

(2) If $Q \geq 1$, we can deduce from Definition 1.3 that $n+Q-1 \leq \tilde{v} \leq n Q$. Thus in our case in Theorem $1.1 \tilde{v}=n+Q-1$ is the smallest. That means the lower bound estimates (1.6) will be optimal.

(3) If $f(\bar{x})=1$ in Theorem 1.1, then $Q=1, \Delta_{X}^{2}=\Delta^{2}$ is the standard bi-harmonic operator. Then $C_{1}(Q)=1, C_{2}(Q)=0$ and $\bar{C}(Q)=\frac{16 \pi^{4} n}{n+4}\left(\frac{\omega_{n-1}|\Omega|_{n}}{n}\right)^{-4 / n}$. Thus the result of Theorem 1.1 will be the same to the result of (1.4) in Levine and Protter [15]. 
In the second part, we shall study the bi-subelliptic operators $\Delta_{X}^{2}$ for more general cases, namely

$$
X=\left(\partial_{x_{1}}, \cdots, \partial_{x_{n-p}}, f_{1}\left(\bar{x}_{(p)}\right) \partial_{x_{n-p+1}}, \cdots, f_{p}\left(\bar{x}_{(p)}\right) \partial_{x_{n}}\right),
$$

where $\bar{x}_{(p)}=\left(x_{1}, \cdots, x_{n-p}\right)$,

$$
f_{j}\left(\bar{x}_{(p)}\right)=\sum_{|\alpha| \leq s_{j}} a_{j \alpha} \bar{x}_{(p)}^{\alpha}, \quad(1 \leq j \leq p<n)
$$

are multivariate polynomials of $\bar{x}_{(p)}$ with order $s_{j}$. Thus $X$ is more general Grushin type degenerate vector fields with $p$ degenerate directions. We suppose that

$\left(H_{2}\right)$ : For each $j, 1 \leq j \leq p<n$, if $f_{j}\left(\bar{x}_{(p)}\right)$ has a unique zero point at origin $\bar{x}_{(p)}=0$ in $\Omega$ only, and there exists a unique multi-index $\alpha_{0 j}$ with $\left|\alpha_{0 j}\right|=s_{0 j} \leq s_{j}$, satisfying $\left.\partial_{\bar{x}_{(p)}}^{\alpha_{0 j}} f_{j}\left(\bar{x}_{(p)}\right)\right|_{\bar{x}_{(p)}=0} \neq$ 0 and $\left.\partial_{\bar{x}_{(p)}}^{\alpha} f_{j}\left(\bar{x}_{(p)}\right)\right|_{\bar{x}_{(p)}=0}=0$ for any $|\alpha|<\left|\alpha_{0 j}\right|$.

Thus we have

Theorem 1.2. Under the condition $\left(H_{2}\right)$ above, the vector fields $X$ satisfies the Hörmander's condition with its Hörmander index $Q=\max \left\{s_{01}, s_{02}, \cdots, s_{0 p}\right\}+1$, and the generalized Métivier index $\tilde{v}=n+\sum_{j=1}^{p} s_{0 j}$. Suppose $\lambda_{j}$ is the $j$-th eigenvalue of the problem (1.1), then for all $k \geq 1$,

$$
\sum_{j=1}^{k} \lambda_{j} \geq \widehat{C}(Q) k^{1+\frac{4}{\bar{\nu}}}-\frac{C_{4}(Q)}{C_{3}(Q)} k
$$

where

$$
\widehat{C}(Q)=\frac{2^{n}}{5 C_{3}(Q) n^{\frac{6+\tilde{v}}{2}}}\left(\frac{\tilde{v}}{\omega_{n-1} \prod_{j=1}^{p}\left(s_{0 j}+1\right)}\right)^{\frac{4+\tilde{v}}{\tilde{v}}}\left(\frac{(2 \pi)^{n}}{|\Omega|_{n}}\right)^{\frac{4}{\tilde{v}}}
$$

where $\tilde{v}=n+\sum_{j=1}^{p} s_{0 j}, C_{3}(Q)$ and $C_{4}(Q)$ are the corresponding sub-elliptic estimate constants in Proposition 2.4, $\omega_{n-1}$ is the area of the unit sphere in $\mathbb{R}^{n},|\Omega|_{n}$ is the volume of $\Omega$.

Remark 1.2. Since $k \lambda_{k} \geq \sum_{j=1}^{k} \lambda_{j}$, then Theorem 1.2 shows that the eigenvalues $\lambda_{k}$ satisfy

$$
\lambda_{k} \geq \widehat{C}(Q) k^{\frac{4}{\bar{v}}}-\frac{C_{4}(Q)}{C_{3}(Q)}, \quad \text { for all } \quad k \geq 1
$$

Our paper is organized as follows. In Section 2, we introduce some preliminaries about subelliptic estimates and discreteness of the Dirichlet eigenvalues for the operator $-\Delta_{X}^{2}$. In Section 3, we prove Theorem 1.1. Finally, we prove Theorem 1.2 in Section 4. 


\section{Preliminaries}

Proposition 2.1. Let the system of vector fields $X=\left(X_{1}, \cdots, X_{m}\right)$ satisfies the Hörmander's condition on $\Omega$ with its Hörmander index $Q \geq 1$, then the following estimate

$$
\left\||\nabla|^{\frac{2}{Q}} u\right\|_{L^{2}(\Omega)}^{2} \leq C(Q)\left\|\Delta_{X} u\right\|_{L^{2}(\Omega)}^{2}+\widetilde{C}(Q)\|u\|_{L^{2}(\Omega)}^{2}
$$

holds for all $u \in C_{0}^{\infty}(\Omega)$, where $\nabla=\left(\partial_{x_{1}}, \cdots, \partial_{x_{m}}\right),|\nabla|^{\frac{2}{\ell}}$ is a pseudo-differential operator with the symbol $|\xi|^{\frac{2}{Q}}$, the constants $C(Q)>0, \widetilde{C}(Q) \geq 0$ depending on $Q$.

Proof. Refer to [12] and [21], the subelliptic operator $\Delta_{X}=\sum_{i=1}^{m} X_{i}^{2}$ satisfies the following sub-elliptic estimate for any $u \in C_{0}^{\infty}(\Omega)$,

$$
\|u\|_{(2 \epsilon)} \leq C_{1}\left\|\Delta_{X} u\right\|_{L^{2}(\Omega)}+C_{2}\|u\|_{L^{2}(\Omega)}
$$

with $\epsilon=\frac{1}{Q}$, where $\|u\|_{(2 \epsilon)}$ is the Sobolev norm of order $2 \epsilon$. On the other hand, we have

$$
\begin{aligned}
\|u\|_{\left(\frac{2}{Q}\right)} & =\left(\int_{n}\left(1+|\xi|^{2}\right)^{\frac{2}{Q}}|\widehat{u}(\xi)|^{2} d \xi\right)^{\frac{1}{2}} \\
& \geq\left(\int_{n}|\xi|^{\frac{4}{Q}}|\widehat{u}(\xi)|^{2} d \xi\right)^{\frac{1}{2}} \\
& =\left\||\nabla|^{\frac{2}{Q}} u\right\|_{L^{2}(n)}=\left\||\nabla|^{\frac{2}{Q}} u\right\|_{L^{2}(\Omega)} .
\end{aligned}
$$

By using the Cauchy-Schwarz inequality we get the following estimate

$$
\left\||\nabla|^{\frac{2}{Q}} u\right\|_{L^{2}(\Omega)}^{2} \leq C(Q)\left\|\Delta_{X} u\right\|_{L^{2}(\Omega)}^{2}+\widetilde{C}(Q)\|u\|_{L^{2}(\Omega)}^{2} .
$$

Thus, we complete the proof.

Proposition 2.2. (cf. $[19,21]$ and [22]) Let the system of vector fields $X=\left(X_{1}, \cdots, X_{m}\right)$ satisfies the Hörmander's condition on $\Omega$, then the operator $\Delta_{X}=\sum_{i=1}^{m} X_{i}^{2}$ is maximally hypo-elliptic, i.e., there exists a constant $C>0$, such that for any $u \in C_{0}^{\infty}(\Omega)$ we have the following maximally hypo-elliptic estimate

$$
\sum_{|\alpha| \leq 2}\left\|X^{\alpha} u\right\|_{L^{2}(\Omega)}^{2} \leq C\left(\left\|\Delta_{X} u\right\|_{L^{2}(\Omega)}^{2}+\|u\|_{L^{2}(\Omega)}^{2}\right),
$$

where $\alpha=\left(\alpha_{1}, \cdots, \alpha_{m}\right)$ is a multi-index with $|\alpha|=\alpha_{1}+\cdots+\alpha_{m}$ and $X^{\alpha}=X_{1}^{\alpha_{1}} \cdots X_{m}^{\alpha_{m}}$. 
Proposition 2.3. Let $X=\left(\partial_{x_{1}}, \cdots, \partial_{x_{n-1}}, f(\bar{x}) \partial_{x_{n}}\right), \bar{x}=\left(x_{1}, x_{2}, \cdots, x_{n-1}\right)$. Here $f(\bar{x})$ is a multivariate polynomial and satisfies the condition $\left(H_{1}\right)$ above. Then $X$ satisfies the Hörmander's condition with its Hörmander index $Q \geq 1$, and we can deduce the following sub-elliptic estimate

$$
\sum_{j=1}^{n-1}\left\|\partial_{x_{j}}^{2} u\right\|_{L^{2}(\Omega)}^{2}+\left\|\left|\partial_{x_{n}}\right| \frac{2}{Q} u\right\|_{L^{2}(\Omega)}^{2} \leq C_{1}(Q)\left\|\Delta_{X} u\right\|_{L^{2}(\Omega)}^{2}+C_{2}(Q)\|u\|_{L^{2}(\Omega)}^{2},
$$

for all $u \in C_{0}^{\infty}(\Omega)$, where $\left|\partial_{x_{n}}\right|^{\frac{2}{Q}}$ is a pseudo-differential operator with the symbol $\left|\xi_{n}\right|^{\frac{2}{Q}}$, $C_{1}(Q)>0, C_{2}(Q) \geq 0$ are constants depending on $Q$.

Proof. From the Plancherel's formula, we have

$$
\begin{aligned}
\left\|\left|\partial_{x_{n}}\right|^{\frac{2}{Q}} u\right\|_{L^{2}(\Omega)}^{2} & =\left\|\left|\xi_{n}\right|^{\frac{2}{Q}} \widehat{u}\right\|_{L^{2}\left(\mathbb{R}^{n}\right)}^{2} \leq\left\||\xi|^{\frac{2}{Q}} \widehat{u}\right\|_{L^{2}\left(\mathbb{R}^{n}\right)}^{2} \\
& =\left\||\nabla|^{\frac{2}{Q}} u\right\|_{L^{2}\left(\mathbb{R}^{n}\right)}^{2}=\left\||\nabla|^{\frac{2}{Q}} u\right\|_{L^{2}(\Omega)}^{2}
\end{aligned}
$$

Also, from the maximally hypo-elliptic estimate of Proposition 2.2 we can deduce that

$$
\sum_{j=1}^{n-1}\left\|\partial_{x_{j}}^{2} u\right\|_{L^{2}(\Omega)}^{2} \leq \sum_{|\alpha| \leq 2}\left\|X^{\alpha} u\right\|_{L^{2}(\Omega)}^{2} \leq C\left(\left\|\Delta_{X} u\right\|_{L^{2}(\Omega)}^{2}+\|u\|_{L^{2}(\Omega)}^{2}\right) .
$$

Combining (2.1), (2.3) and (2.4) we can deduce that

$$
\sum_{j=1}^{n-1}\left\|\partial_{x_{j}}^{2} u\right\|_{L^{2}(\Omega)}^{2}+\left\|\left|\partial_{x_{n}}\right|^{\frac{2}{Q}} u\right\|_{L^{2}(\Omega)}^{2} \leq C_{1}(Q)\left\|\Delta_{X} u\right\|_{L^{2}(\Omega)}^{2}+C_{2}(Q)\|u\|_{L^{2}(\Omega)}^{2} .
$$

Thus, we complete the proof.

Proposition 2.4. Let $X=\left(\partial_{x_{1}}, \cdots, \partial_{x_{n-p}}, f_{1}\left(\bar{x}_{(p)}\right) \partial_{x_{n-p+1}}, \cdots, f_{p}\left(\bar{x}_{(p)}\right) \partial_{x_{n}}\right), \quad \bar{x}_{(p)}=$ $\left(x_{1}, x_{2}, \cdots, x_{n-p}\right)$. Here $f_{j}\left(\bar{x}_{(p)}\right)$ (for $\left.1 \leq j \leq p<n\right)$ are multivariate polynomials which satisfying the condition $(H 2)$ above. Then $X$ satisfies the Hörmander's condition with its Hörmander index $Q \geq 1$, and we get the following sub-elliptic estimate

$$
\sum_{i=1}^{n-p}\left\|\partial_{x_{i}}^{2} u\right\|_{L^{2}(\Omega)}^{2}+\sum_{j=1}^{p}\left\|\left|\partial_{x_{n-p+j}}\right|^{\frac{2}{s_{0 j}+1}} u\right\|_{L^{2}(\Omega)}^{2} \leq C_{3}(Q)\left\|\Delta_{X} u\right\|_{L^{2}(\Omega)}^{2}+C_{4}(Q)\|u\|_{L^{2}(\Omega)^{\prime}}^{2}
$$

for all $u \in C_{0}^{\infty}(\Omega)$, where $\left|\partial_{x_{j}}\right|^{\frac{2}{r}}$ is a pseudo-differential operator with the symbol $\left|\xi_{j}\right|^{\frac{2}{r}}$, and the constants $C_{3}(Q)>0, C_{4}(Q) \geq 0$ depending on $Q$. 
Proof. We consider the system of vector fields $\tilde{X}=\left(\partial_{x_{1}}, \cdots, \partial_{x_{n-p}}, f_{j}\left(\bar{x}_{(p)}\right) \partial_{x_{n-p+j}}\right)($ for $1 \leq$ $j \leq p<n)$ defined on the projection $\Omega_{x_{j}^{\prime}}$ of $\Omega$ on the direction $x_{j}^{\prime}=\left(x_{1}, \cdots, x_{n-p}, x_{n-p+j}\right)$. Similar to Proposition 2.3, for all $j(1 \leq j \leq p)$, we have

$$
\sum_{i=1}^{n-p}\left\|\partial_{x_{i}}^{2} u\right\|_{L^{2}\left(\Omega_{x_{j}^{\prime}}\right)}^{2}+\left\|\mid \partial_{x_{n-p+j}}{ }^{\frac{2}{s_{j}+1}} u\right\|_{L^{2}\left(\Omega_{x_{j}^{\prime}}\right)}^{2} \leq \widehat{C}_{1}(Q)\left\|\Delta_{\tilde{X}} u\right\|_{L^{2}\left(\Omega_{x_{j}^{\prime}}\right)}^{2}+\widehat{C}_{2}(Q)\|u\|_{L^{2}\left(\Omega_{x_{j}^{\prime}}\right.}^{2} .
$$

Then for all $j(1 \leq j \leq p)$, we have

$$
\sum_{i=1}^{n-p}\left\|\partial_{x_{i}}^{2} u\right\|_{L^{2}(\Omega)}^{2}+\left\|\left|\partial_{x_{n-p+j}}\right|^{\frac{2}{s_{0 j}+1}} u\right\|_{L^{2}(\Omega)}^{2} \leq \widehat{C}_{1}(Q)\left\|\Delta_{\tilde{X}} u\right\|_{L^{2}(\Omega)}^{2}+\widehat{C}_{2}(Q)\|u\|_{L^{2}(\Omega)}^{2} .
$$

By using the Cauchy-Schwarz inequality and Proposition 2.2, there exists a constant $C_{3}>0$ such that

$$
\left\|\Delta_{\tilde{X}} u\right\|_{L^{2}(\Omega)}^{2} \leq C_{3} \sum_{|\alpha| \leq 2}\left\|X^{\alpha} u\right\|_{L^{2}(\Omega)}^{2} \leq C_{3} C\left(\left\|\Delta_{X} u\right\|_{L^{2}(\Omega)}^{2}+\|u\|_{L^{2}(\Omega)}^{2}\right)
$$

where $C$ is given in Proposition 2.2. Finally, we get the following sub-elliptic estimate from (2.6)

$$
\sum_{i=1}^{n-p}\left\|\partial_{x_{i}}^{2} u\right\|_{L^{2}(\Omega)}^{2}+\sum_{j=1}^{p}\left\|\left|\partial_{x_{n-p+j}}\right|^{\frac{2}{s_{j}+1}} u\right\|_{L^{2}(\Omega)}^{2} \leq C_{3}(Q)\left\|\Delta_{X} u\right\|_{L^{2}(\Omega)}^{2}+C_{4}(Q)\|u\|_{L^{2}(\Omega)}^{2} .
$$

Thus, we complete the proof.

Next, for the Dirichlet eigenvalues problem (1.1), we have

Proposition 2.5. The Dirichlet eigenvalues problem (1.1) has a sequence of discrete eigenvalues $\left\{\lambda_{j}\right\}_{j \geq 1}$, which satisfying $0<\lambda_{1} \leq \lambda_{2} \leq \lambda_{3} \leq \cdots \leq \lambda_{k} \cdots$ and $\lambda_{k} \rightarrow+\infty$ as $k \rightarrow+\infty$. Also, the corresponding eigenfunctions $\left\{\phi_{k}(x)\right\}_{k \geq 1}$ constitute an orthonormal basis of $L^{2}(\Omega)$ and an orthogonal basis of $H_{X, 0}^{2}(\Omega)$.

The proof of Proposition 2.5 depends the following lemma:

Lemma 2.1. If $u \in H_{X, 0}^{2}(\Omega)$, then for $1 \leq j \leq m, X_{j} u \in H_{X, 0}^{1}(\Omega)$.

Proof. Since $u \in H_{X, 0}^{2}(\Omega)$, we have $X_{i}\left(X_{j} u\right) \in L^{2}(\Omega)$ for any $1 \leq i, j \leq m$, and $\left(X_{j} u\right) \in L^{2}(\Omega)$. That implies $X_{j} u \in H_{X}^{1}(\Omega)$. Now, $u \in H_{X, 0}^{2}(\Omega)$, then there exists a sequence $\varphi_{i} \in C_{0}^{\infty}(\Omega)$ which converges to $u$ in $H_{X, 0}^{2}(\Omega)$. That means $X_{j} \varphi_{i} \rightarrow X_{j} u$ in $H_{X}^{1}(\Omega)$. Observe that $X_{j} \varphi_{i} \in$ $H_{X, 0}^{1}(\Omega)$ and $H_{X, 0}^{1}(\Omega)$ is a Hilbert space, thus we have $X_{j} u \in H_{X, 0}^{1}(\Omega)$. 
Proof of Proposition 2.5. We know that the definition domain of $\Delta_{X}^{2}$ is

$$
\operatorname{dom}\left(\Delta_{X}^{2}\right)=\left\{u \in H_{X, 0}^{2}(\Omega) \mid \Delta_{X}^{2} u \in L^{2}(\Omega)\right\} .
$$

Thus, for $X_{j}$ to be formally skew-adjoint, then for any function $u \in C_{0}^{\infty}(\Omega)$ and $v \in$ $\operatorname{dom}\left(\Delta_{X}^{2}\right)$, we have

$$
\begin{aligned}
\int_{\Omega} u \Delta_{X}^{2} v d x & =\int_{\Omega} v \Delta_{X}^{2} u d x \\
& =\int_{\Omega} v \Delta_{X}\left(\Delta_{X} u\right) d x=\sum_{j=1}^{m} \int_{\Omega} v \cdot X_{j}^{2}\left(\Delta_{X} u\right) d x
\end{aligned}
$$

Since $v \in H_{X, 0}^{2} \subset H_{X, 0}^{1}(\Omega)$, and from the result of Lemma 2.1, $X_{j} v \in H_{X, 0}^{1}(\Omega)$. Then the equation above gives

$$
\int_{\Omega} u \Delta_{X}^{2} v d x=-\sum_{j=1}^{m} \int_{\Omega} X_{j} v \cdot X_{j}\left(\Delta_{X} u\right) d x=\sum_{j=1}^{m} \int_{\Omega} X_{j}^{2} v \cdot\left(\Delta_{X} u\right) d x,
$$

that gives the following Green formula:

$$
\int_{\Omega} u \Delta_{X}^{2} v d x=\int_{\Omega} \Delta_{X} u \cdot \Delta_{X} v d x, \quad \text { for } u \in H_{X, 0}^{2}(\Omega), \quad v \in \operatorname{dom}\left(\Delta_{X}^{2}\right) .
$$

On the other hand, for $u \in H_{X, 0}^{2}(\Omega)$,

$$
\|u\|_{H_{X}^{2}}^{2}=\|u\|_{L^{2}(\Omega)}^{2}+\sum_{i=1}^{m}\left\|X_{i} u\right\|_{L^{2}(\Omega)}^{2}+\sum_{i, j=1}^{m}\left\|X_{i} X_{j} u\right\|_{L^{2}(\Omega)}^{2} .
$$

Thus we have

$$
\|u\|_{H_{X}^{2}} \geq\|u\|_{L^{2}(\Omega)}+\sum_{j=1}^{m}\left\|X_{j}^{2} u\right\|_{L^{2}(\Omega)} \geq\left\|\Delta_{X} u\right\|_{L^{2}(\Omega)} .
$$

By maximally hypoellipticity of $\Delta_{X}$ (also see Proposition 2.2 above), we have following estimate for any $u \in H_{X, 0}^{2}(\Omega)$,

$$
\|u\|_{H_{X}^{2}}^{2}=\sum_{|\alpha| \leq 2}\left\|X^{\alpha} u\right\|_{L^{2}(\Omega)}^{2} \leq C\left(\left\|\Delta_{X} u\right\|_{L^{2}(\Omega)}^{2}+\|u\|_{L^{2}(\Omega)}^{2}\right) .
$$

Furthermore, the Poincaré inequality gives

$$
\|u\|_{L^{2}(\Omega)}^{2} \leq C_{1}\|X u\|_{L^{2}(\Omega)}^{2} \leq C_{1}\left|\left(\Delta_{X} u, u\right)\right| \leq C_{1}\left\|\Delta_{X} u\right\|_{L^{2}(\Omega)} \cdot\|u\|_{L^{2}(\Omega)} .
$$

Thus for any $0<\epsilon<1$ there is $C_{\epsilon}>0$, such that

$$
\left\|\Delta_{X} u\right\|_{L^{2}(\Omega)} \cdot\|u\|_{L^{2}(\Omega)} \leq C_{\epsilon}\left\|\Delta_{X} u\right\|_{L^{2}(\Omega)}^{2}+\epsilon\|u\|_{L^{2}(\Omega)}^{2} .
$$


That means from (2.9) that there exists $C_{2}>0$, such that

$$
\|u\|_{H_{X}^{2}}^{2} \leq C_{2}\left\|\Delta_{X} u\right\|_{L^{2}(\Omega)}^{2} .
$$

Hence from (2.8) and (2.10) one has for any $u \in H_{X, 0}^{2}(\Omega)$,

$$
\left\|\Delta_{X} u\right\| \leq\|u\|_{H_{X}^{2}} \leq C_{3}\left\|\Delta_{X} u\right\| .
$$

Thus we define that

$$
[u, \varphi]=\left(\Delta_{X} u, \Delta_{X} \varphi\right),
$$

then $[\cdot, \cdot]$ is another inner product, and $H_{X, 0}^{2}(\Omega)$ with this inner product is complete.

Now, we choose $u, v \in \operatorname{dom}\left(\Delta_{X}^{2}\right)$, then

$$
\left(\Delta_{X}^{2} u, v\right)=\left(\Delta_{X} u, \Delta_{X} v\right)=\left(\Delta_{X}^{2} v, u\right) .
$$

Hence, $\Delta_{X}^{2}$ is symmetric operator in $\operatorname{dom}\left(\Delta_{X}^{2}\right)$. Also

$$
\left(\Delta_{X}^{2} u, u\right)=\left(\Delta_{X} u, \Delta_{X} u\right) \geq 0,
$$

which implies that $\Delta_{X}^{2}$ is positive in $\operatorname{dom}\left(\Delta_{X}^{2}\right)$.

Next, for any given $f \in L^{2}(\Omega)$ and any $\varphi \in H_{X, 0}^{2}(\Omega)$, we define a functional $f(\varphi)=(f, \varphi)$. Since

$$
|(f, \varphi)| \leq\|f\|_{L^{2}(\Omega)} \cdot\|\varphi\|_{L^{2}(\Omega)} \leq\|f\|_{L^{2}(\Omega)} \cdot\|\varphi\|_{H_{X}^{2}(\Omega)},
$$

then the functional $(f, \varphi)$ is a continuous linear functional on Hilbert space $H_{X, 0}^{2}(\Omega)$. By Riesz representation theorem, there exists a unique $u \in H_{X, 0}^{2}(\Omega)$ such that

$$
(f, \varphi)=[u, \varphi]=\left(\Delta_{X} u, \Delta_{X} \varphi\right) .
$$

Thus the Green formula (2.7) gives that

$$
\left(\Delta_{X}^{2} u, \varphi\right)=\left(\Delta_{X} u, \Delta_{X} \varphi\right)=(f, \varphi)
$$

holds for any $\varphi \in C_{0}^{\infty}(\Omega)$. That implies $\Delta_{X}^{2} u=f$, i.e., $u \in \operatorname{dom}\left(\Delta_{X}^{2}\right)$. This proves the existence of the resolvent operator $R:=\left(\Delta_{X}^{2}\right)^{-1}$, and $R f=u$.

On the other hand, if we choose $\varphi=u$ in (2.13), then $(R f, f)=(u, f)=\left\|\Delta_{X} u\right\|_{L^{2}(\Omega)}^{2} \geq 0$. $R$ is positive in $L^{2}(\Omega)$. Meanwhile we have

$$
\|R f\|_{L^{2}(\Omega)}^{2}=\|u\|_{L^{2}(\Omega)}^{2} \leq C\|f\|_{L^{2}(\Omega)}\|R f\|_{L^{2}(\Omega)},
$$

this implies that $R$ is bounded in $L^{2}(\Omega)$. In order to prove the operator $R$ is self-adjoint, it suffices to prove that $R$ is symmetric, i.e.,

$$
(R f, g)=(f, R g) \text { for all } f, g \in L^{2}(\Omega) .
$$


Let $R f=u, R g=v$, and choosing $\varphi=v$ in (2.13), we obtain

$$
\left(\Delta_{X} u, \Delta_{X} v\right)=(f, R g) .
$$

Since the left hand side is symmetric in $u$ and $v$, we conclude that the right side is symmetric in $f$ and $g$. That implies that $R$ is symmetric. Also, we know that the operator $R^{-1}:=\Delta_{X}^{2}$ is a self-adjoint on $\operatorname{dom}\left(\Delta_{X}^{2}\right)$.

Similarly, we can prove that the inverse operator $\left(\Delta_{X}^{2}+\alpha \cdot i d\right)^{-1}$ exists and is bounded for any $\alpha \geq 0$. We see that $-\alpha$ is a regular value of $\Delta_{X}^{2}$, hence $\operatorname{spec}\left(\Delta_{X}^{2}\right) \subset(0,+\infty)$. Moreover, we can deduce that $R: L^{2}(\Omega) \rightarrow H_{X, 0}^{2}(\Omega)$ is continuous, this is because that

$$
\|R f\|_{H_{X}^{2}}^{2} \leq C\left(\left\|\Delta_{X}(R f)\right\|_{L^{2}(\Omega)}^{2}\right) \leq C(f, R f) \leq C\|f\|_{L^{2}(\Omega)}\|R f\|_{L^{2}(\Omega)} \leq C\|f\|_{L^{2}(\Omega)}\|R f\|_{H_{X}^{2}(\Omega)} .
$$

By using the subelliptic estimate, we know that $H_{X, 0}^{2}$ can be continuously embedded into the standard Sobolev space $H^{\frac{2}{Q}}(\Omega)$, and $H^{\frac{2}{Q}}(\Omega)$ can be compactly embedded into $L^{2}(\Omega)$. Hence $R$ is a compact operator from $L^{2}(\Omega)$ to $L^{2}(\Omega)$. By spectral theory we know that $R$ has positive discrete eigenvalues $\mu_{i}, \mu_{1} \geq \mu_{2} \geq \cdots \geq \mu_{k} \geq \cdots$ and $\mu_{k} \rightarrow 0$ as $k \rightarrow+\infty$; and the corresponding eigenfunctions $\phi_{i}$ of $R$ form an orthonormal basis of $L^{2}(\Omega)$, namely

$$
R \phi_{i}=\mu_{i} \phi_{i}
$$

That means the eigenfunctions $\left\{\phi_{i}\right\}_{i \geq 1}$ will be the orthogonal basis of $H_{X, 0}^{2}(\Omega)$. Finally we let $\lambda_{i}=\mu_{i}^{-1}$, then $\lambda_{i}$ are the Dirichlet eigenvalues of $\Delta_{X}^{2}$ which will be discrete and satisfying $0<\lambda_{1} \leq \lambda_{2} \leq \cdots \leq \lambda_{k} \leq \cdots$, and $\lambda_{k} \rightarrow+\infty$ as $k \rightarrow+\infty$. The proof of Proposition 2.5 is completed.

\section{Proof of Theorem 1.1}

Lemma 3.1 (cf. $[3,16])$. For the system of vector fields $X=\left(X_{1}, \cdots, X_{m}\right)$, if $\left\{\phi_{j}\right\}_{j=1}^{k}$ are the set of orthonormal eigenfunctions corresponding to the eigenvalues $\left\{\lambda_{j}\right\}_{j=1}^{k}$. Define

$$
\Phi(x, y)=\sum_{j=1}^{k} \phi_{j}(x) \phi_{j}(y)
$$

Then for $\widehat{\Phi}(z, y)=(2 \pi)^{-n / 2} \int_{\mathbb{R}^{n}} \Phi(x, y) e^{-i x \cdot z} d x$ to be the partial Fourier transformation of $\Phi(x, y)$ with respect to the $x$-variable, we have

$$
\int_{\Omega} \int_{\mathbb{R}^{n}}|\widehat{\Phi}(z, y)|^{2} d z d y=k \quad \text { and } \quad \int_{\Omega}|\widehat{\Phi}(z, y)|^{2} d y \leq(2 \pi)^{-n}|\Omega|_{n} .
$$


Lemma 3.2 (cf. [8]). Let $f$ be a real-valued function defined on $\mathbb{R}^{n}$ with $0 \leq f \leq M_{1}$, and for $Q \in \mathbb{N}^{+}$,

$$
\int_{\mathbb{R}^{n}}\left(\sum_{j=1}^{n-1} z_{j}^{2}+\left|z_{n}\right|^{\frac{2}{Q}}\right)^{2} f(z) d z \leq M_{2}
$$

Then

$$
\int_{\mathbb{R}^{n}} f(z) d z \leq \frac{\left(Q M_{1} \omega_{n-1}\right)^{\frac{4}{n+Q+3}}}{n+Q-1}\left(\frac{n(n+Q+3)}{A_{Q}}\right)^{\frac{n+Q-1}{n+Q+3}} M_{2}^{\frac{n+Q-1}{n+Q+3}},
$$

where $\omega_{n-1}$ is the area of the unit sphere in $\mathbb{R}^{n}$, and

$$
A_{Q}= \begin{cases}\min \left\{1, n^{\frac{3-Q}{2}}\right\}, & Q \geq 2, \\ n, & Q=1 .\end{cases}
$$

Proof of Theorem 1.1. From the results of Proposition 2.5, let $\left\{\lambda_{k}\right\}_{k \geq 1}$ be a sequence of the eigenvalues for the problem (1.1), and $\left\{\phi_{k}(x)\right\}_{k \geq 1}$ be the corresponding eigenfunctions, then $\left\{\phi_{k}(x)\right\}_{k \geq 1}$ constitute an orthogonal basis of $H_{X, 0}^{2}(\Omega)$.

Let

$$
\Phi(x, y)=\sum_{j=1}^{k} \phi_{j}(x) \phi_{j}(y)
$$

by Cauchy-Schwarz inequality we have

$$
\begin{aligned}
& \int_{\mathbb{R}^{n}} \int_{\Omega}\left(\sum_{j=1}^{n-1} z_{j}^{2}+\left|z_{n}\right|^{\frac{2}{Q}}\right)^{2}|\widehat{\Phi}(z, y)|^{2} d y d z \\
\leq & n \int_{\mathbb{R}^{n}} \int_{\Omega}\left(\sum_{j=1}^{n-1} z_{j}^{4}+\left|z_{n}\right|^{\frac{4}{Q}}\right)|\widehat{\Phi}(z, y)|^{2} d y d z .
\end{aligned}
$$

Next, by using integration-by-parts, we have

$$
\begin{aligned}
\sum_{j=1}^{k} \lambda_{j} & =\sum_{j=1}^{k} \int_{\Omega} \lambda_{j} \phi_{j}(x) \cdot \phi_{j}(x) d x=\sum_{j=1}^{k} \int_{\Omega} \Delta_{X}^{2} \phi_{j}(x) \cdot \phi_{j}(x) d x \\
& =\sum_{j=1}^{k} \int_{\Omega} X\left(\Delta_{X} \phi_{j}(x)\right) \cdot X \phi_{j}(x) d x=\sum_{j=1}^{k} \int_{\Omega} \Delta_{X} \phi_{j}(x) \cdot \Delta_{X} \phi_{j}(x) d x \\
& =\int_{\Omega} \int_{\Omega} \sum_{j=1}^{k}\left|\Delta_{X} \phi_{j}(x) \phi_{j}(y)\right|^{2} d x d y=\int_{\Omega} \int_{\Omega}\left|\Delta_{X} \Phi(x, y)\right|^{2} d x d y .
\end{aligned}
$$


Then by using Plancherel's formula and Proposition 2.3, we have

$$
\begin{aligned}
& \int_{\mathbb{R}^{n}} \int_{\Omega}\left(\sum_{j=1}^{n-1} z_{j}^{2}+\left|z_{n}\right|^{\frac{2}{Q}}\right)^{2}|\widehat{\Phi}(z, y)|^{2} d y d z \\
\leq & n \int_{\mathbb{R}^{n}} \int_{\Omega}\left(\sum_{j=1}^{n-1} z_{j}^{4}+\left|z_{n}\right|^{\frac{4}{Q}}\right)|\widehat{\Phi}(z, y)|^{2} d y d z \\
= & n \int_{n} \int_{\Omega}\left(\sum_{j=1}^{n-1}\left|\partial_{x_{j}}^{2} \Phi(x, y)\right|^{2}+\left.\left.|| \partial_{x_{n}}\right|^{\frac{2}{Q}} \Phi(x, y)\right|^{2}\right) d y d x \\
= & n \int_{\Omega} \int_{\Omega}\left(\sum_{j=1}^{n-1}\left|\partial_{x_{j}}^{2} \Phi(x, y)\right|^{2}+\left.\left.|| \partial_{x_{n}}\right|^{\frac{2}{Q}} \Phi(x, y)\right|^{2}\right) d y d x \\
\leq & n\left[C_{1}(Q) \int_{\Omega} \int_{\Omega}\left|\Delta_{X} \Phi(x, y)\right|^{2} d x d y+C_{2}(Q) \int_{\Omega} \int_{\Omega}|\Phi(x, y)|^{2} d x d y\right] .
\end{aligned}
$$

Thus from (3.2) and Lemma 3.1 above, we can deduce that

$$
\int_{n} \int_{\Omega}\left(\sum_{j=1}^{n-1} z_{j}^{2}+\left|z_{n}\right|^{\frac{2}{Q}}\right)^{2}|\widehat{\Phi}(z, y)|^{2} d y d z \leq n\left(C_{1}(Q) \sum_{j=1}^{k} \lambda_{j}+C_{2}(Q) k\right) .
$$

Next, we choose

$$
f(z)=\int_{\Omega}|\widehat{\Phi}(z, y)|^{2} d y, \quad M_{1}=(2 \pi)^{-n}|\Omega|_{n}, \quad M_{2}=n\left(C_{1}(Q) \sum_{j=1}^{k} \lambda_{j}+C_{2}(Q) k\right) .
$$

Then from the result of Lemma 3.2, we know that for any $k \geq 1$,

$$
\leq \frac{Q \omega_{n-1}(2 \pi)^{-n}|\Omega|_{n}}{n+Q-1}\left(\frac{n(n+Q+3)}{(2 \pi)^{-n}|\Omega|_{n} Q A_{Q} \omega_{n-1}}\right)^{\frac{n+Q-1}{n+Q+3}}\left(n\left(C_{1}(Q) \sum_{j=1}^{k} \lambda_{j}+C_{2}(Q) k\right)\right)^{\frac{n+Q-1}{n+Q+3}} .
$$

This means, for any $k \geq 1$,

$$
\sum_{j=1}^{k} \lambda_{j} \geq \widetilde{C}(Q) k^{1+\frac{4}{\bar{t}}}-\frac{C_{2}(Q)}{C_{1}(Q)} k
$$

with

$$
\widetilde{C}(Q)=\frac{A_{Q}}{C_{1}(Q) n^{2}(n+Q+3)}\left(\frac{(2 \pi)^{n}}{Q \omega_{n-1}|\Omega|_{n}}\right)^{\frac{4}{n+Q-1}}(n+Q-1)^{\frac{n+Q+3}{n+Q-1}} .
$$

The proof of Theorem 1.1 is completed. 


\section{Proof of Theorem 1.2}

Lemma 4.1. Let $f$ be a real-valued function defined on $\mathbb{R}^{n}$ with $0 \leq f \leq M_{1}$, and for $p, q \in \mathbb{N}^{+}$,

$$
\int_{\mathbb{R}^{n}}\left(\sum_{i=1}^{n-p} z_{i}^{2}+\sum_{j=1}^{p}\left|z_{n-p+j}\right|^{\frac{2}{s_{0 j}+1}}\right)^{2} f(z) d z \leq M_{2}
$$

Then

$$
\int_{\mathbb{R}^{n}} f(z) d z \leq \frac{\omega_{n-1} \prod_{j=1}^{p}\left(s_{0 j}+1\right)}{\tilde{v}}\left(\frac{5 n^{\frac{4+\tilde{v}}{2}}}{2^{n}}\right)^{\frac{\tilde{v}}{4+\tilde{v}}} M_{1}^{\frac{4}{4+\tilde{v}}} M_{2}^{\frac{\tilde{v}}{4+\tilde{v}}},
$$

where $\tilde{v}=n+\sum_{j=1}^{p} s_{0 j}, \omega_{n-1}$ is the area of the unit sphere in $\mathbb{R}^{n}$.

Proof. First, we choose $R$ such that

$$
\int_{\mathbb{R}^{n}}\left(\sum_{i=1}^{n-p} z_{i}^{2}+\sum_{j=1}^{p}\left|z_{n-p+j}\right|^{\frac{2}{s_{0 j}+1}}\right)^{2} g(z) d z=M_{2}
$$

where

$$
g(z)= \begin{cases}M_{1}, & \sum_{i=1}^{n-p} z_{i}^{2}+\sum_{j=1}^{p}\left|z_{n-p+j}\right|^{\frac{2}{s_{0 j}+1}} \leq R^{2}, \\ 0, & \sum_{i=1}^{n-p} z_{i}^{2}+\sum_{j=1}^{p}\left|z_{n-p+j}\right|^{\frac{2}{s_{0 j}+1}}>R^{2} .\end{cases}
$$

Then

$$
\left[\left(\sum_{i=1}^{n-p} z_{i}^{2}+\sum_{j=1}^{p}\left|z_{n-p+j}\right|^{\frac{2}{s_{j}+1}}\right)^{2}-R^{4}\right](f(z)-g(z)) \geq 0
$$

Hence we have

$$
R^{4} \int_{\mathbb{R}^{n}}(f(z)-g(z)) d z \leq \int_{\mathbb{R}^{n}}\left(\sum_{i=1}^{n-p} z_{i}^{2}+\sum_{j=1}^{p}\left|z_{n-p+j}\right|^{\frac{2}{s_{0 j}+1}}\right)^{2}(f(z)-g(z)) d z \leq 0 .
$$

That means

$$
\int_{\mathbb{R}^{n}} f(z) d z \leq \int_{\mathbb{R}^{n}} g(z) d z
$$

Now we have

$$
M_{2}=\int_{\mathbb{R}^{n}}\left(\sum_{i=1}^{n-p} z_{i}^{2}+\sum_{j=1}^{p}\left|z_{n-p+j}\right|^{\frac{2}{s_{0 j}+1}}\right)^{2} g(z) d z=M_{1} \int_{\widetilde{B}_{R}}\left(\sum_{i=1}^{n-p} z_{i}^{2}+\sum_{j=1}^{p}\left|z_{n-p+j}\right|^{\frac{2}{s_{0 j}+1}}\right)^{2} d z,
$$


where

$$
\widetilde{B}_{R}=\left\{z \in \mathbb{R}^{n}, \quad \sum_{i=1}^{n-p} z_{i}^{2}+\sum_{j=1}^{p}\left|z_{n-p+j}\right|^{\frac{2}{s_{0 j}+1}} \leq R^{2}\right\} .
$$

Next, we change the variables as follows,

$$
z_{i}=z_{i}^{\prime} \quad(i=1,2, \cdots, n-p), \quad z_{n-p+j}=\operatorname{sgn}\left(z_{n-p+j}^{\prime}\right)\left|z_{n-p+j}^{\prime}\right|^{s_{0 j}+1}, \quad(j=1,2, \cdots, p) .
$$

Then we have the following determinant of Jacobian,

$$
\left|\operatorname{det}\left(\frac{\partial\left(z_{1}, \cdots, z_{n}\right)}{\partial\left(z_{1}^{\prime}, \cdots, z_{n}^{\prime}\right)}\right)\right|=\prod_{j=1}^{p}\left(s_{0 j}+1\right)\left|z_{n-p+j}^{\prime}\right|^{s_{0 j}} .
$$

Hence

$$
\begin{aligned}
M_{2} & =M_{1} \int_{\widetilde{B}_{R}}\left(\sum_{i=1}^{n-p} z_{i}^{2}+\sum_{j=1}^{p}\left|z_{n-p+j}\right|^{\frac{2}{s_{0 j}+1}}\right)^{2} d z \\
& =M_{1} \prod_{j=1}^{p}\left(s_{0 j}+1\right) \int_{B_{R}}|z|^{4} \prod_{j=1}^{p}\left|z_{n-p+j}\right|^{s_{0 j}} d z \\
& \geq M_{1} \prod_{j=1}^{p}\left(s_{0 j}+1\right) \int_{A_{R}}|z|^{4} \prod_{j=1}^{p}\left|z_{n-p+j}\right|^{s_{0 j}} d z,
\end{aligned}
$$

where

$$
B_{R}=\left\{z \in \mathbb{R}^{n},|z| \leq R\right\}, \quad A_{R}=\left\{z \in \mathbb{R}^{n},\left|z_{j}\right| \leq \frac{R}{\sqrt{n}}, j=1, \cdots, n\right\} .
$$

By a direct calculation, we have

$$
\begin{aligned}
& \int_{A_{R}}|z|^{4} \prod_{j=1}^{p}\left|z_{n-p+j}\right|^{s_{0 j}} d z \\
& \geq \int_{A_{R}}\left|z_{1}\right|^{4} \prod_{j=1}^{p}\left|z_{n-p+j}\right|^{s_{0 j}} d z \\
& =2 \int_{0}^{\frac{R}{\sqrt{n}}}\left|z_{1}\right|^{4} d z_{1} \times \prod_{j=1}^{p}\left(2 \int_{0}^{\frac{R}{\sqrt{n}}}\left|z_{n-p+j}\right|^{s_{0 j}} d z_{n-p+j}\right) \times\left(2 \int_{0}^{\frac{R}{\sqrt{n}}} 1 d z\right)^{n-p-1} \\
& =\frac{2^{n}}{5} \frac{1}{\prod_{j=1}^{p}\left(s_{0 j}+1\right)} n^{-\frac{n+4+\sum_{j=1}^{p} s_{0 j}}{2}} R^{n+4+\sum_{j=1}^{p} s_{0 j}}=\frac{2^{n}}{5} \frac{1}{\prod_{j=1}^{p}\left(s_{0 j}+1\right)} n^{-\frac{4+\tilde{v}}{2}} R^{4+\tilde{v}} .
\end{aligned}
$$

Then we have

$$
M_{2} \geq \frac{2^{n} M_{1}}{5} n^{-\frac{4+\tilde{v}}{2}} R^{4+\tilde{v}}
$$


From the definition of $g(z)$, we know that

$$
\begin{aligned}
\int_{\mathbb{R}^{n}} g(z) d z & =M_{1} \int_{\widetilde{B}_{R}} d z=M_{1} \prod_{j=1}^{p}\left(s_{0 j}+1\right) \int_{B_{R}} \prod_{j=1}^{p}\left|z_{n-p+j}\right|^{s_{0 j}} d z \\
\leq & M_{1} \prod_{j=1}^{p}\left(s_{0 j}+1\right) \int_{B_{R}}|z|^{\sum_{j=1}^{p} s_{0 j}} d z=M_{1} \prod_{j=1}^{p}\left(s_{0 j}+1\right) \int_{0}^{R} \omega_{n-1} r^{n-1+\sum_{j=1}^{p} s_{0 j}} d r \\
& =\frac{M_{1} \omega_{n-1} \prod_{j=1}^{p}\left(s_{0 j}+1\right)}{n+\sum_{j=1}^{p} s_{0 j}} R^{n+\sum_{j=1}^{p} s_{0 j}}=\frac{M_{1} \omega_{n-1} \prod_{j=1}^{p}\left(s_{0 j}+1\right)}{\tilde{v}} R^{\tilde{v}} .
\end{aligned}
$$

From (4.1), (4.2) and (4.3), we obtain

$$
\int_{\mathbb{R}^{n}} f(z) d z \leq \int_{\mathbb{R}^{n}} g(z) d z \leq \frac{\omega_{n-1} \prod_{j=1}^{p}\left(s_{0 j}+1\right)}{\tilde{v}}\left(\frac{5 n^{\frac{4+\tilde{v}}{2}}}{2^{n}}\right)^{\frac{\tilde{v}}{4+\tilde{v}}} M_{1}^{\frac{4}{4+\tilde{v}}} M_{2}^{\frac{\tilde{v}}{4+\tilde{v}}}
$$

where $\tilde{v}=n+\sum_{j=1}^{p} s_{0 j}$. Lemma 4.1 is proved.

Proof of Theorem 1.2. Let $\left\{\lambda_{k}\right\}_{k \geq 1}$ be a sequence of the eigenvalues for the problem (1.1), $\left\{\phi_{k}(x)\right\}_{k \geq 1}$ be the corresponding eigenfunctions. Then $\left\{\phi_{k}(x)\right\}_{k \geq 1}$ constitute an orthogonal basis of $H_{X, 0}^{2}(\Omega)$.

Let $\Phi(x, y)=\sum_{j=1}^{k} \phi_{j}(x) \phi_{j}(y)$. Thus, by using the Cauchy-Schwarz inequality, we have

$$
\begin{array}{r}
\int_{\mathbb{R}^{n}} \int_{\Omega}\left(\sum_{j=1}^{n-p} z_{j}^{2}+\sum_{j=1}^{p}\left|z_{n-p+j}\right|^{\frac{2}{s_{0 j}+1}}\right)^{2}|\widehat{\Phi}(z, y)|^{2} d y d z \\
\leq n \int_{\mathbb{R}^{n}} \int_{\Omega}\left(\sum_{j=1}^{n-p} z_{j}^{4}+\sum_{j=1}^{p}\left|z_{n-p+j}\right|^{\frac{4}{s_{0 j}+1}}\right)|\widehat{\Phi}(z, y)|^{2} d y d z .
\end{array}
$$

Similar to the result of (3.2), we obtain that

$$
\sum_{j=1}^{k} \lambda_{j}=\int_{\Omega} \int_{\Omega}\left|\Delta_{X} \Phi(x, y)\right|^{2} d x d y .
$$

Then by using Plancherel's formula and Proposition 2.4, we have

$$
\begin{aligned}
& \int_{\mathbb{R}^{n}} \int_{\Omega}\left(\sum_{j=1}^{n-p} z_{j}^{2}+\sum_{j=1}^{p}\left|z_{n-p+j}\right|^{\frac{2}{s_{0 j}+1}}\right)^{2}|\widehat{\Phi}(z, y)|^{2} d y d z \\
\leq & n \int_{\mathbb{R}^{n}} \int_{\Omega}\left(\sum_{j=1}^{n-p} z_{j}^{4}+\sum_{j=1}^{p}\left|z_{n-p+j}\right|^{\frac{4}{s_{0 j}+1}}\right)|\widehat{\Phi}(z, y)|^{2} d y d z
\end{aligned}
$$




$$
\begin{aligned}
& =n \int_{\mathbb{R}^{n}} \int_{\Omega}\left(\sum_{j=1}^{n-p}\left|\partial_{x_{j}}^{2} \Phi(x, y)\right|^{2}+\left.\left.\sum_{j=1}^{p}|| \partial_{x_{n-p+j}}\right|^{\frac{2}{s_{0 j}+1}} \Phi(x, y)\right|^{2}\right) d y d x \\
& =n \int_{\Omega} \int_{\Omega}\left(\sum_{j=1}^{n-p}\left|\partial_{x_{j}}^{2} \Phi(x, y)\right|^{2}+\left.\left.\sum_{j=1}^{p}|| \partial_{x_{n-p+j}}\right|^{\frac{2}{s_{0 j}+1}} \Phi(x, y)\right|^{2}\right) d y d x \\
& \leq n\left[C_{3}(Q) \int_{\Omega} \int_{\Omega}\left|\Delta_{X} \Phi(x, y)\right|^{2} d x d y+C_{4}(Q) \int_{\Omega} \int_{\Omega}|\Phi(x, y)|^{2} d x d y\right] .
\end{aligned}
$$

Thus from (4.5) and Lemma 3.1 above, we can deduce that

$$
\int_{\mathbb{R}^{n}} \int_{\Omega}\left(\sum_{j=1}^{n-p} z_{j}^{2}+\sum_{j=1}^{p}\left|z_{n-p+j}\right|^{\frac{2}{s_{0 j}+1}}\right)^{2}|\widehat{\Phi}(z, y)|^{2} d y d z \leq n\left(C_{3}(Q) \sum_{j=1}^{k} \lambda_{i}+C_{4}(Q) k\right) .
$$

Finally, we choose

$$
f(z)=\int_{\Omega}|\widehat{\Phi}(z, y)|^{2} d y, \quad M_{1}=(2 \pi)^{-n}|\Omega|_{n}, \quad M_{2}=n\left(C_{3}(Q) \sum_{i=1}^{k} \lambda_{i}+C_{4}(Q) k\right) .
$$

Then from the Lemma 4.1, we have for any $k \geq 1$,

$$
k \leq \frac{\omega_{n-1} \prod_{j=1}^{p}\left(s_{0 j}+1\right)}{\tilde{v}}\left((2 \pi)^{-n}|\Omega|_{n}\right)^{\frac{4}{4+\tilde{v}}}\left(\frac{5 n^{\frac{4+\tilde{v}}{2}}}{2^{n}}\right)^{\frac{\tilde{v}}{4+\tilde{v}}}\left(n\left(C_{3}(Q) \sum_{j=1}^{k} \lambda_{j}+C_{4}(Q) k\right)\right)^{\frac{\tilde{v}}{4+\tilde{v}}}
$$

This means, for any $k \geq 1$,

$$
\sum_{j=1}^{k} \lambda_{j} \geq \widehat{C}(Q) k^{1+\frac{4}{\bar{v}}}-\frac{C_{4}(Q)}{C_{3}(Q)} k
$$

where $\tilde{v}=n+\sum_{j=1}^{p} s_{0 j}$, and

$$
\widehat{C}(Q)=\frac{2^{n}}{5 C_{3}(Q) n^{\frac{6+\tilde{v}}{2}}}\left(\frac{\tilde{v}}{\omega_{n-1} \prod_{j=1}^{p}\left(s_{0 j}+1\right)}\right)^{\frac{4+\tilde{v}}{\tilde{\hat{v}}}}\left(\frac{(2 \pi)^{n}}{|\Omega|_{n}}\right)^{\frac{4}{\overline{\hat{\sigma}}}} .
$$

The proof of Theorem 1.2 is completed.

\section{Acknowledgements}

The authors would like to thank the referee for the suggestions. Also, this work is supported by National Natural Science Foundation of China (Grants Nos. 11631011 and 11626251). 


\section{References}

[1] Agmon, S., On kernels, eigenvalues and eigenfunctions of operators related to elliptic problems, Commun. Pure Appl. Math., 18 (1965), 627-663.

[2] Bramanti, M., An Invitation to Hypoelliptic Operators and Hörmander's Vector Fields, Springer, New York, (2014).

[3] Chen, H. and Luo, P., Lower bounds of Dirichlet eigenvalues for some degenerate elliptic operators, Calc. Var. Partial Differ. Eqs., 54 (2015), 2831-2852.

[4] Chen, H., Luo, P. and Tian, S., Lower bounds of Dirichlet eigenvalues for degenerate elliptic operators and degenerate Schrödinger operators, Max-Planck-Institut für Mathematik in den Naturwissenschaften Leipzig, (2013).

[5] Chen, H., Liu, X. and Wei, Y., Dirichlet problem for semilinear edge-degenerate elliptic equations with singular potential term, J. Differ. Eqs., 252 (2012), 4289-4314.

[6] Chen, H., Qiao, R., Luo, P. and Xiao, D., Lower and upper bounds of Dirichlet eigenvalues for totally characteristic degenerate elliptic operators, Sci. China Math., 57 (2014), 2235-2246.

[7] Cheng, Q. M., Wei, G. X., Upper and lower bounds for eigenvalues of the clamped plate problem, arxiv:1201.6103v1 [math.DG] (2012).

[8] Chen, H. and Zhou, Y. F., Lower bounds of eigenvalues for a class of bi-subelliptic operators, J. Differ. Eqs., 262 (2017), 5860-5879.

[9] Fefferman, C. and Phong, D., Subelliptic eigenvalue problems. In: Proceedings of the Conference on Harmonic Analysis in Honor of Antoni Zygmund, Wadsworth Math. Series, 590606 (1981).

[10] Hansson, A. M. and Laptev, A., Sharp Spectral Inequalities for the Heisenberg Laplacian, LMS Lectore Note Series (354), 100-115, Cambridge University Press, Cambridge (2008).

[11] Hörmander, L., Hypoelliptic second order differential equations, Acta Math., 119 (1967), 147-171.

[12] Jerison, D. and Sánchez-Calle, A., Subelliptic second order differential operators, Complex Analysis III (1987).

[13] Jerison, D. and Sánchez-Calle, A., Estimates for the heat kernel for sum of squares of vector fields, Indiana Univ. Math. J., 35 (1986), 835-854.

[14] Kohn, J. J., Subellipticity of $\bar{\partial}-$ Neumann problem on pseudoconvex domains: sufficient conditions, Acta Math., 142 (1979), 79-122.

[15] Levine, H. A. and Protter, M. H., Unrestricted lower bounds for eigenvalues for classes of elliptic equations and systems of equations with applications to problems in elasticity, Math. Meth. Appl. Sci., 7 (1985), 210-222.

[16] Li, P. and Yau, S. T., On the Schrödinger equation and the eigenvalue problem, Commun. Math. Phys., 88 (1983), 309-318.

[17] Métivier, G., Fonction spectrale d'oérateurs non elliptiques, Commun. PDE., 1 (1976), 467519.

[18] Morimoto, Y. and Xu, C. J., Logarithmic Sobolev inequality and semi-linear Dirichlet problems for infinitely degenerate elliptic operators, Astérisque, 234 (2003), 245-264.

[19] Nier, F. and Helffer, B., Hypoelliptic estimates and spectral theory for Fokker-Planck operators and Witten Laplacians, Springer Science \& Business Media, (2005).

[20] Pleijel, A., On the eigenvalues and eigenfunctions of elastic plates, Commun. Pure Appl. Math., 3 (1950), 1-10.

[21] Rothschild, L. P. and Stein, L. P., Hypoelliptic differential operators and nilpotent groups. Acta Math., 137 (1977), 248-315. 
[22] Rothschild, L. P., A criterion for hypoellipticity of operators constructed from vector fields, Commun. PDE., 4(6) (1979), 645-699.

[23] Xu, C. J., Regularity problem for quasi-linear second order subelliptic equations, Commun. Pure Appl. Math., 45 (1992), 77-96. 